\title{
FORMULATION AND IN VITRO EVALUATION OF METHAZOLAMIDE ELASTIC VESICULAR SYSTEMS
}

\author{
Rania R. Elazreg ${ }^{a, b}$, Mahmoud E. Soliman ${ }^{a}$, Abdelhameed EI Shamy ${ }^{a}$, Samar \\ Mansour $^{a}$ \\ ${ }^{a}$ Pharmaceutics and Industrial Pharmacy Department, Faculty of Pharmacy, \\ Ain Shams University, Cairo, Egypt \\ ${ }^{b}$ Department of Pharmaceutics, Faculty of Pharmacy, Zawia University, Libya
}

\section{ABSTRACT}

Eye is the most unique organ of the body. Various drug delivery systems are used to deliver drug into the eyes but there are various limitations of conventional systems, so various novel approaches like nanovesicular systems were used to improve ocular contact time, bioavailability, residence time and dosing frequency. Nanovesicular system with elastic properties can provide a controlled delivery of Methazolamide (MZA) which is used to treat glaucoma by inhibiting the action of carbonic anhydrase enzyme. The elastic nanovesicular carriers were formulated using Span 60 (SP 60) with one of the following edge activators (EA): Tween 80 (TW80), Tween 60 (TW 60), Brij 35 (BJ 35), and Brij 58 (BJ 58) in the ratio (90:10; 80:20; 70:30 w: w). The prepared formulae were evaluated for their sizes, entrapment efficiencies (EE \%), relative deformability, and in vitro release. The results show that the selected formulae (S1, S4, S7, and S8) have the suitable particle size and EE\%, thus can be used for any further investigations.

Keywords: elastic vesicles, Methazolamide, Edge Activators, Carbonic anhydrase inhibitors.

\section{INTRODUCTION}

Eye contains several protection mechanisms which decrease ocular drug absorption topically and lead to short duration of action. These mechanisms mainly involve the drainage of tear fluid with blinking of the eye leading to about 10 -fold reduction in the intraocular concentration of drug (Lang, 1995). Among other reasons which may also cause low drug absorption are the limited corneal permeability and the passage of drug via nasolacrimal duct. Therefore, the ocular bioavailability of topically applied drug solutions is not more than 1-5\% (Jarvinen et al., 1995).

To overcome the previously mentioned obstacles which may face ocular drug delivery, several approaches have been used like the vesicular systems (Kaur et al., 2004).

Methazolamide (MZA) is a weakly acidic sulfonamide derivative which is slightly soluble in water. It is indicated for the treatment of glaucoma by inhibiting the action of carbonic anhydrase enzyme. However, MZA was found to have some serious side effects when taken orally such as allergic reactions (e.g. difficulty of breathing, swollen lips, tongue or face), bleeding, and tremors in the hands or feet. Therefore, the topical administration of MZA to the eye is more preferred in order to reduce these side effects. However, the poor aqueous solubility of MZA $\left(\sim 1.7 \mathrm{mg} / \mathrm{ml}\right.$ at $\left.25^{\circ} \mathrm{C}\right)$ (Maren et al., 1977) , as well as its low corneal permeability $\left(1.75 \times 10^{-4} \mathrm{~cm} /\right.$ hour) (Edelhauser and Maren, 1988) make its topical delivery inefficient. Different technique have been utilized to formulate MZA in a form that can be delivered topically e.g. cyclodextrin eye drops (Fridriksdóttir et al., 1997), calcium phosphate nanoparticles (Chen et al., 2010), in situ- gels (Qian et al., 2010), cationic nanostructured heterolipid matrices (Youshia $\boldsymbol{e t}$ 
al., 2012), solid lipid nanoparticles which was prepared by modified emulsion-solvent evaporation (Li et al., 2011).

Moreover, elastic vesicular systems that consist of a mixture of span60 together with different edge activators, can be used to increase the drug residence time and consequently its ocular bioavailability (Kakkar and Kaur, 2011).

The aim of the work in this paper was to prepare and characterize Methazolamide loaded elastic vesicular system.

\section{EXPERIMENTAL}

\section{Materials}

Methazolamide (MZA) was purchased from Jiaxing Taixing chemical and Pharma Co, Itd (Jiaxing, China). Brij 35, Brij 58, Span 60, Tween 60, and Tween 80 were purchased from Sigma Chemical Co., St. Louis, USA. Disodium hydrogen Phosphate, ethanol 99\% pure absolute, potassium dihydrogen phosphate, and sodium chloride were purchased from Adwic, El-Nasr pharmaceutical CO. (Cairo, Egypt). Spectra/Por dialysis membrane, 12,000-14,000 molecular weight cut off was purchased from Spectrum Laboratories Inc., Rancho Dominguez, Canada.

\section{Equipment}

Centrifuge (Herml, Z 216MK centrifug, Gosheim,Germany), particle size analyzer NanoZS (Malvern instruments, Malvern, UK), Rotary evaporator (Janke and Kunkel, IKA Laboratories, Germany), USP dissolution apparatus (Pharma test, Hainburg, Germany), and UV- Visible spectrophotometer (model UV -1601pc; shimadzu,Kyoto, Japan)

\section{Methodology}

\section{1- Preparation of MZA-loaded elastic vesicular system}

Elastic vesicular system containing Span 60 and edge activators (EAs) (namely Brij 35, Brij 58, Tween 60, and Tween 80) were prepared by ethanol injection method using different weight ratios of Span60: EA (90:10; 80:20; 70:30 w: w). Briefly, Span 60 and calculated amount of MZA were dissolved in $4 \mathrm{ml}$ of ethanol to produce final concentration of $0.05 \%(\mathrm{w} / \mathrm{v})$, and then injected into magnetically stirred aqueous solution of the EA (Kakkar and Kaur, 2011). The ethanol was evaporated by Rotary evaporator, and the formed milky vesicular dispersions were used for further investigations.

\section{2- Determination of MZA entrapment efficiency (EE \%) in elastic vesicular system}

The EE\% of MZA in elastic vesicular system was determined by measuring the concentration of free drug in the vesicular dispersion. The non-encapsulated MZA was separated by centrifugation using Nanosep ${ }^{\circledR}$. A definite volume of the prepared elastic vesicular system was transferred to the upper chamber of the Nanosep ${ }^{\circledR}$, elastic vesicular system solutions were centrifuged at $5000 \mathrm{rpm}$ at $4^{\circ} \mathrm{C}$ for 15 minute. The amount of free drug in the filtrate was determined spectrophotometrically at 290nm using a UV-Visible spectrophotometer.

The EE\% was calculated according to the following equation as follows:

$$
\mathrm{EE} \%=\left[\frac{W t-W f}{W t}\right]_{\times 100}
$$

Where Wt is the total amount of drug used in the formulation and $\mathrm{Wf}$ is the amount of free drug remaining in the supernatant.

\section{3- Particle size (PS) and zeta potential (ZP) analysis of elastic vesicular system}


The PS and ZP of elastic vesicular system were determined at $25^{\circ} \mathrm{C}$ using particle size analyzer NanoZS (Guinedi et al., 2005). Size and charge measurement using this device is based on Dynamic Light Scattering (DLS). Samples were transferred to disposable polystyrene cells or disposable plain folded capillary zeta cells, and all measurements were performed in triplicate.

\section{4- Measurement of elasticity of the vesicles (Relative deformability)}

The measurement of elasticity (Relative deformability) of the elastic vesicular system loaded with MZA solution was carried out by extrusion technique (Van den Bergh et al., 1999, 2001; El Zaafarany et al., 2010) through a locally fabricated stainless steel pressure filter holder. The vesicles were extruded through membrane filters with pore size of $220 \mathrm{~nm}$ at constant pressure of 0.248 Bar. The experiments were carried out in triplicates and relative deformability as an indicator for elasticity was calculated according to the following equation (Salama et al., 2012):-

$$
\left.\mathrm{D}=\frac{j}{t} \frac{r v}{(r p}\right)^{2}
$$

Where $\mathrm{D}$ is the deformability index $(\mathrm{ml} / \mathrm{s}) ; \mathrm{j}$ is the amount of suspension which is extruded (ml); $t$ is the time of extrusion (s); rv is the size of vesicles after extrusion $(\mathrm{nm})$; and $\mathrm{rp}$ is the pore size of the barrier $(\mathrm{nm})$.

\section{5- In vitro release of MZA from elastic vesicular system}

In vitro drug release was done by dialysis membrane diffusion technique using USP dissolution apparatus fitted with glass cylinder $(10 \mathrm{~cm}$ in length and $2.5 \mathrm{~cm}$ in diameter). The cylinders were fixed in the device shaft from one end using clips while the other end was covered by cellulose membrane (Spectra/Por dialysis membrane 12, 000-14,000 molecular weight cut off) to retain elastic vesicular system while allowing the free drug to be released into the dissolution medium in the vessels (Nasr et al., 2008; Hathout et al., 2007). Accurately measured amounts of elastic MZA vesicular system equivalent to $1 \mathrm{mg}$ MZA were transferred to the glass cylinder, and the cylinder was immersed in $50 \mathrm{ml}$ dissolution media phosphate-buffered saline (PBS) adjusted at PH (7.4) for Methazolamide elastic vesicular system (Attama et al., 2008).

The dissolution media was kept at $37^{\circ} \mathrm{C} \pm 0.5$ while the cylinders were rotated at $50 \mathrm{rpm} .1 \mathrm{ml}$ samples were withdrawn at different time intervals $(0.25,0.5,0.75,1,1.5$, $2,4,6,8$ hours) and replaced with $1 \mathrm{ml}$ of fresh medium, then assayed spectrophotometrically at $290 \mathrm{~nm}$. All the results presented in the release studies are the mean values of three runs.

\section{6- Determination of drug release kinetics}

To know the mechanism of drug release from the selected formulae, the data were treated according to first-order (log cumulative percentage of drug remaining vs. time), Higuchi's (cumulative percentage of drug released vs. square root of time), zero order (cumulative amount of drug released vs. time) pattern, and Korsmeyer-Peppas model (plotted as log cumulative percentage drug release vs. log time) (Dash, et al., 2010; Prabhu, et al., 2010).

\section{7- Physical stability studies}

\section{A- Determination of the percent of retained MZA in elastic vesicles after storage}

The stability study was carried out by investigation the ability of the selected elastic vesicles formulae (S1, S4, S7, and S8) to retain the drug (drug retention 
behavior). The elastic vesicles formulation was kept at refrigeration temperature (4$8^{\circ} \mathrm{C}$ ), throughout the study, elastic vesicles formulations were stored in sealed glass vials. The samples were withdrawn at different time intervals zero time, after three month, and six month. The drug leakage from the formulations was analyzed spectrophotometrically as previously described in section (2) (Patidar and Jain 2012; Shahiwala and Misra 2002).

\section{B- Vesicle size analysis}

Aggregation or fusion of the vesicles as a function of time was determined by measuring the changes in vesicles diameters for the selected elastic vesicles formulae (S1, S4, S7, and S8), after stored in sealed glass vials at temperature $\left(4-8^{\circ} \mathrm{C}\right)$, at time intervals zero time, after three month, and six month using (DLS) technique as previously mentioned in section (3) (Patidar and Jain 2012).

\section{RESULTS AND DISCUSSION}

Nanovesicular elastic systems were prepared using ethanol injection method. Different ratios of Span 60 to edge activators were tested in order to choose the formulae which show the highest encapsulation efficiency along with small size to be used for further investigations. Data shown in Table (1) reveals that the percentage of MZA entrapped in the prepared elastic vesicular systems were in the range of 23 to $94 \%$.

Generally, all tested formulae (S1-S12) showed high EE\% values. The high values for EE\% could be due to the high transition temperature (Tc) of Span 60. It was previously reported that Span 60 showed high EE\% compared to the unsaturated Span 80 when tested for the entrapment of various drugs (Yoshioka et al., 1994; Lingan MA et al., 2011; Abdallah et al., 2013; Akhilesh et al., 2012).

Statistical analysis showed the effect of Span 60: EA ratio on EE\%, since the difference between formulae S1, and S2 was not significant. However, when edge activator (EA) content increased to $30 \%$, the EE\% decreased to $68.36 \%$. While for formulae S4, S5, S6, S7, S8 and S9 there were no significant difference between the EE\% values, when the content of EA was 10, 20, and 30\%. However, for S10, S11, and S12 which contained Brij 58, we observed significant decrease in EE\% with the increase in EA content $(\mathrm{P}<0.05)$.

It was previously found that the increase in the content of EA result in poreformation of vesicular bilayer until the concentration reaches a certain threshold. When, such threshold is reached vesicles based on micelles or mixed micelles start to form, leading to a decrease in EE\% (Van den Bergh et al., 2001).

All formulations in Table 1 show a mean Particle size (PS) less than $1 \mu \mathrm{m}$ in diameter. As we screened the size of different formulae we observed that S1, S4, S7, and S8 showed the smallest size and high drug entrapment values when compared to other vesicular formulations. The size of S1, S4, S7, and S8 ranged from 270 to $329 \mathrm{~nm}$; hence they were selected for further investigations.

Generally, the use of edge activators with lower HLB resulted in vesicles with smaller size (Yoshioka et al., 1994; Basha et al., 2013). The relationship observed between vesicle size and surfactant HLB could be due to the decrease in surface energy of vesicles, prepared by these surfactants. Vesicles prepared using low HLB surfactants are previously reported to have smaller size compared to those prepared by high HLB surfactants (El Zaafarany et al., 2010; Akhilesh et al., 2012) 
Thus, upon comparing the PS of formulae (S1, S4, S7, and S8), we found that the smallest PS was for S1composed of Span 60 and Tween 60. The variation in PS noticed between different formulae can be explained by the difference in HLB values of their EAs components. The HLB values for different EAs are 14.9, 15, and 16.9 for TW60, TW80, and Bj 35 respectively. Among the different EAs; TW60 showed the lowest HLB value and smallest PS when measured by (DLS).

For Brij 35 (formulae S7 and S8) as EA, it was noticed that by increasing the ratio of EA from 10 to $20 \%$ the PS of vesicles decreased from 315.53 to $279.133 \mathrm{~nm}$.

The zetapotential (ZP) values of all prepared elastic vesicular system were in negative region ranging from -12.86 to $-30.66 \mathrm{mV}$. The negative charge on the surface of elastic vesicular system is responsible for forming a shield that prevent vesicles from aggregation and impart colloidal stability (Wilson et al., 2008; Huang et al., 2011) . The recorded values for Tween 80 elastic vesicular system were formed to be near the values recorded by (Basha et al., 2013) which was in the range from -23 to $-29 \mathrm{mV}$ for vesicles of similar components to those in our study.

Elasticity is an important character of elastic vesicular formulations, which can squeeze to pass through pores of biological membranes and decrease the risk of vesicle disrupted while passing through membrane (El Zaafarany et al., 2010). This property differentiates elastic vesicles from other vesicular systems.

The elasticity can be expressed by relative deformability. Fig 1 reveals the average deformability of formulae (S1, S4, and S8). It demonstrates that all formulae have elastic characters. A special properties for Tween 80 is highly flexible and non bulky hydrocarbon chains (El Zaafarany et al., 2010). in addition to the unsaturated alkyl chain (double bond) which render vesicles prepared using Tween 80 more membrane permeable (Abdallah et al., 2013).

Fig 2 shows the in vitro drug release profile of MZA solution, and selected formulae (S1, S4, S7 and S8). It was observed that MZA solution released approximately $100 \%$ of drug within $4 \mathrm{hrs}$, it was significantly higher than all tested elastic formulae $(\mathrm{P}<0.05)$. Elastic vesicular formulations S1, S4, S7 and S8 showed $90.31 \%, 97.64 \%, 77.22 \%$ and $95.53 \%$ drug release respectively in $8 \mathrm{hrs}$. The result of in vitro drug release profile of the formulations showed that elastic vesicular formulations provides more prolonged release of MZA when compared to MZA solution.

Table 2 shows that formulae S4, and S7 followed zero order release kinetics. This indicated that the drug release was independent of concentration of drug entrapped (Prabhu, et al., 2010). However the elastic vesicles S1, and S8 released MZA by diffusion controlled mechanism. This may be due to the formation of a core enriched with active compound leading to a membrane release governed by Fick's law of diffusion (Muller, et al., 2002). The difference between S7, and S8 in the kinetic of release can be due to the increase in EA content wich leads to the increase in the tendency of pore-formation in vesicle bilayers and hence their permeability of MZA from vesicles lead to the change in release mechanism (Basha, et al., 2010). The value of $n$ characterizes the release mechanism of drug as described in Table 2. They were higher than 0.89 for all formulae which indicate that the systems follow Super case II mechanism (Dash, et al., 2010).

The stability results for MZA retained in elastic vesicles formulae (S1, S4, S7, and S8) after 180 days, are demonstrated in Fig 3. Statistical analysis shows that there is a significant difference between the values of $\mathrm{EE} \%$ for freshly prepared vesicles and after storing the vesicles for a period of 90 and 180 days for all tested formulae except S7 which didn't show any significant difference after a period of 90 days $(\mathrm{P}<0.05)$. 
The effect of storage on the mean particle size of elastic vesicles formulae (S1, S4, S7, and S8) is demonstrated in Fig 4. The results showed significant increase in the particle size of the stored formulae after periods 90 and 180 days, with exception of S8 which didn't show any significant change in size when measured after period of 90 days $(\mathrm{P}<0.05)$. These results may be due to the fusion and aggregation of the vesicles after storage (Padamwar and pokharkar 2006).

Table 1. The composition of different elastic vesicular system loaded with MZA preparations, Entrapment Efficiency, Particle size and Zeta potential (mean \pm SD)

\begin{tabular}{|c|c|c|c|c|c|}
\hline $\begin{array}{c}\text { Zeta } \\
\text { potential } \\
(\mathrm{mV}) \pm \mathrm{SD}\end{array}$ & $\begin{array}{c}\text { Mean } \\
\text { Particle size } \\
(\mathrm{nm}) \pm \mathrm{SD}\end{array}$ & $\begin{array}{c}\text { Entrapment } \\
\text { Efficiency } \\
\text { Percent } \\
\text { EE\% } \pm \text { SD }\end{array}$ & $\begin{array}{c}\text { Weight } \\
\text { Ratio }\end{array}$ & $\begin{array}{c}\text { Formulae } \\
\text { Composition }\end{array}$ & $\begin{array}{c}\text { Formulae } \\
\text { Code }\end{array}$ \\
\hline$-16.3 \pm 0.7$ & $276.26 \pm 2.77$ & $82.80 \pm 0.65$ & $90: 10$ & Sp60+ Tw60 & S1 \\
\hline$-15.6 \pm 1.53$ & $704.93 \pm 8.64$ & $81.99 \pm 0.18$ & $80: 20$ & Sp60 \pm Tw60 & S2 \\
\hline$-14.76 \pm 1.07$ & $585.1 \pm 4.46$ & $68.36 \pm 0.24$ & $70: 30$ & Sp60+ Tw60 & S3 \\
\hline$-27.7 \pm 1.15$ & $328.56 \pm 3.19$ & $81.74 \pm 0.43$ & $90: 10$ & Sp60+ Tw80 & S4 \\
\hline$-24.93 \pm 0.51$ & $761.06 \pm 6.15$ & $80.89 \pm 2.20$ & $80: 20$ & Sp60+ Tw80 & S5 \\
\hline$-30.66 \pm 0.49$ & $657.53 \pm 6.40$ & $78.69 \pm 2.32$ & $70: 30$ & Sp60+ Tw80 & S6 \\
\hline$-19.73 \pm 0.37$ & $315.53 \pm 1.56$ & $94.19 \pm 0.13$ & $90: 10$ & Sp60+ BJ35 & S7 \\
\hline$-23.03 \pm 0.68$ & $279.13 \pm 2.45$ & $93.69 \pm 0.15$ & $80: 20$ & Sp60+ BJ35 & S8 \\
\hline$-14 \pm 0.45$ & $995.66 \pm 3.05$ & $91.94 \pm 0.16$ & $70: 30$ & Sp60+ BJ35 & S9 \\
\hline$-19.43 \pm 0.51$ & $630.46 \pm 7.59$ & $68.76 \pm 0.26$ & $90: 10$ & Sp60+ BJ58 & S10 \\
\hline$-21.33 \pm 1.52$ & $531.63 \pm 3.09$ & $27.76 \pm 0.04$ & $80: 20$ & Sp60+ BJ58 & S11 \\
\hline$-12.86 \pm 0.77$ & $533.46 \pm 8.30$ & $23.29 \pm 1.95$ & $70: 30$ & Sp60 + BJ58 & S12 \\
\hline
\end{tabular}

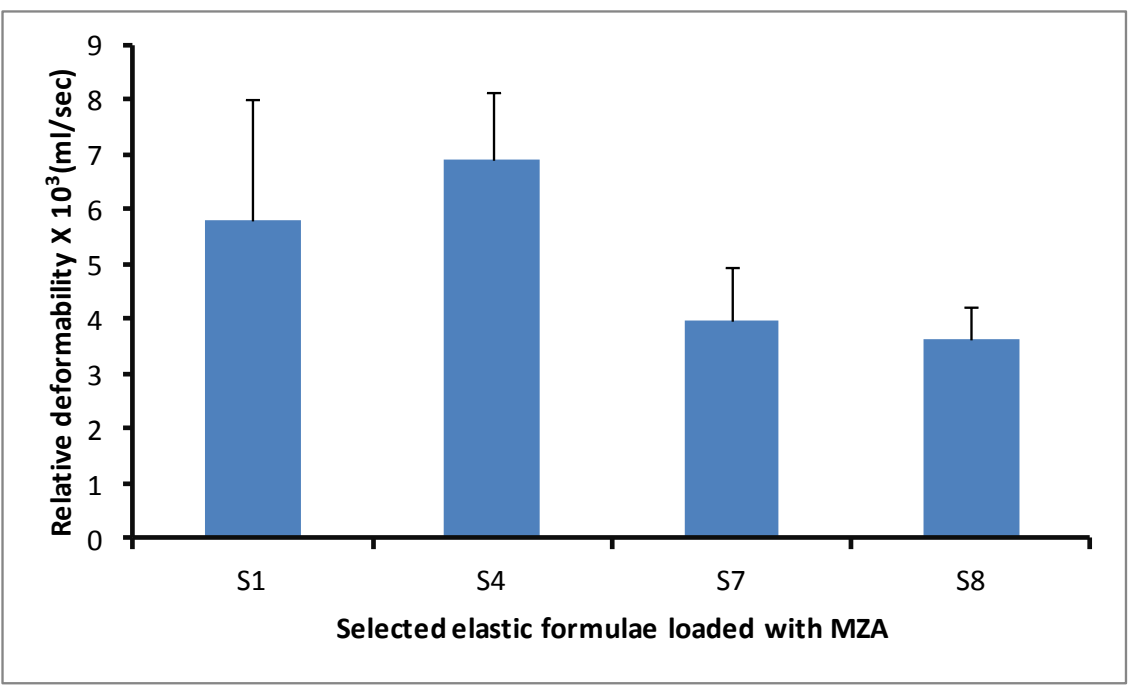

Fig 1: Average of relative deformability $(\mathrm{ml} / \mathrm{sec}) * 10^{3}$ of elastic vesicular system loaded with MZA 


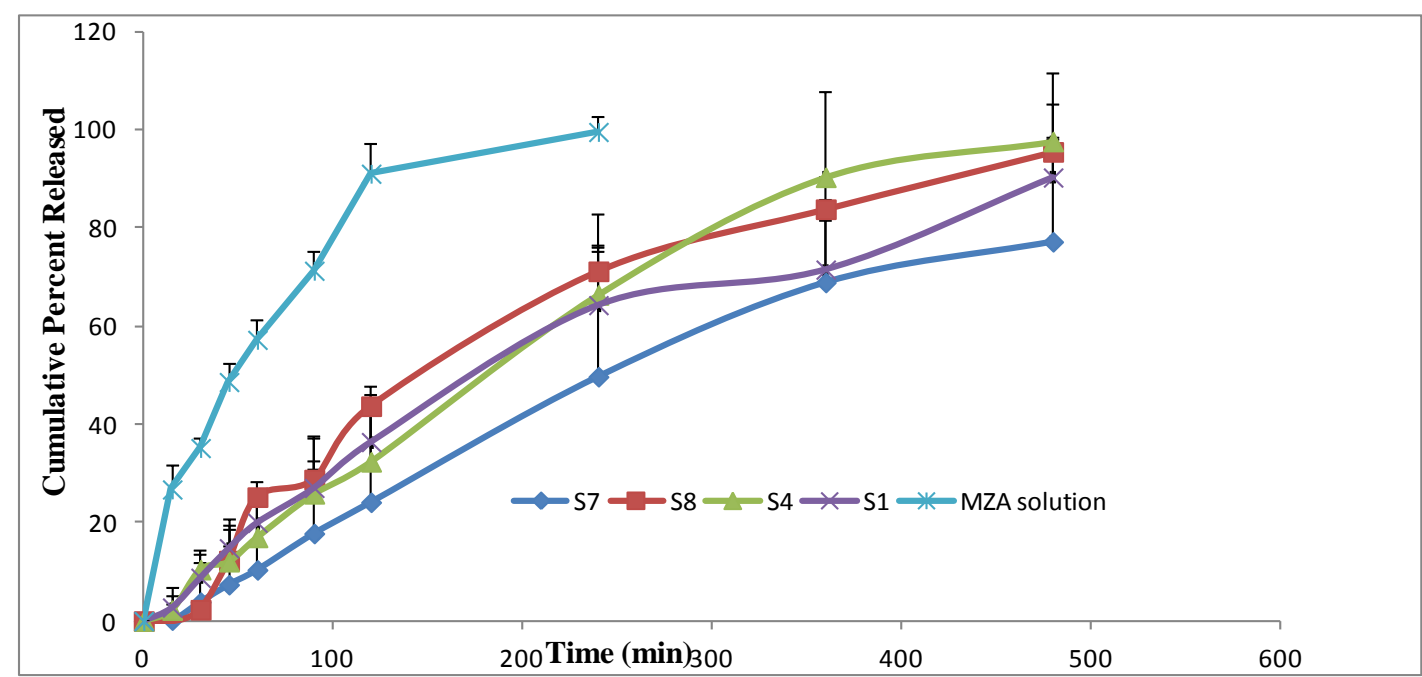

Fig 2: Release profiles of MZA from elastic vesicles solution in PBS at $37^{\circ} \mathrm{C}$

Table 2. Kinetic parameter of the release data of MZA from elastic vesicles

\begin{tabular}{|c|c|c|c|c|c|}
\hline \multirow[b]{2}{*}{$\begin{array}{l}\text { Order of } \\
\text { release }\end{array}$} & \multirow[b]{2}{*}{$\begin{array}{l}\text { Peppas } \\
\text { model (n) }\end{array}$} & \multicolumn{3}{|c|}{$\mathrm{r}^{2}$} & \multirow[b]{2}{*}{$\begin{array}{l}\text { Formulae } \\
\text { Code }\end{array}$} \\
\hline & & $\begin{array}{l}\text { Higuchi } \\
\text { model }\end{array}$ & First order & Zero order & \\
\hline Diffusion & 1.104 & 0.992 & 0.443 & 0.989 & S1 \\
\hline Zero order & 1.140 & 0.988 & 0.678 & 0.992 & $\mathrm{~S} 4$ \\
\hline Zero order & 1.148 & 0.985 & 0.234 & 0.994 & S7 \\
\hline Diffusion & 1.484 & 0.989 & 0.601 & 0.982 & S8 \\
\hline
\end{tabular}

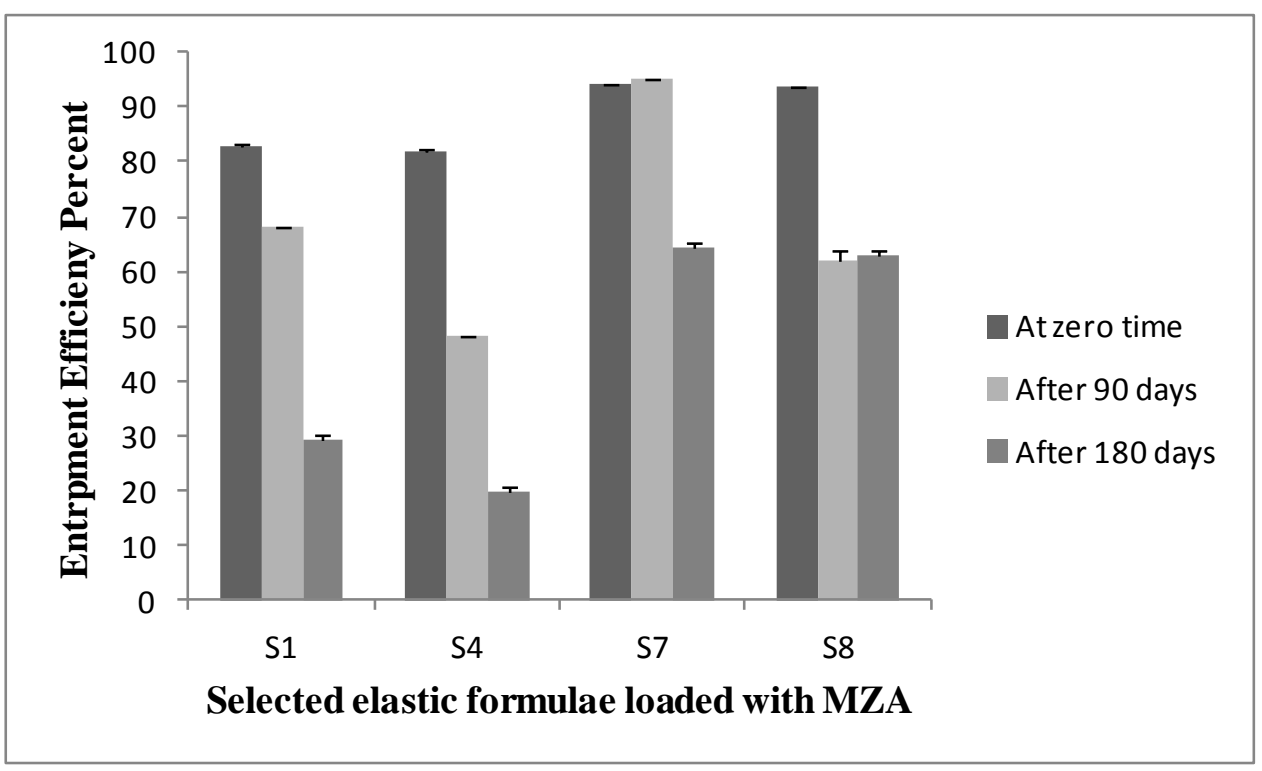

Fig 3: Percent MZA retained in elastic vesicles after interval of time at $4^{\circ} \mathrm{C}$ 


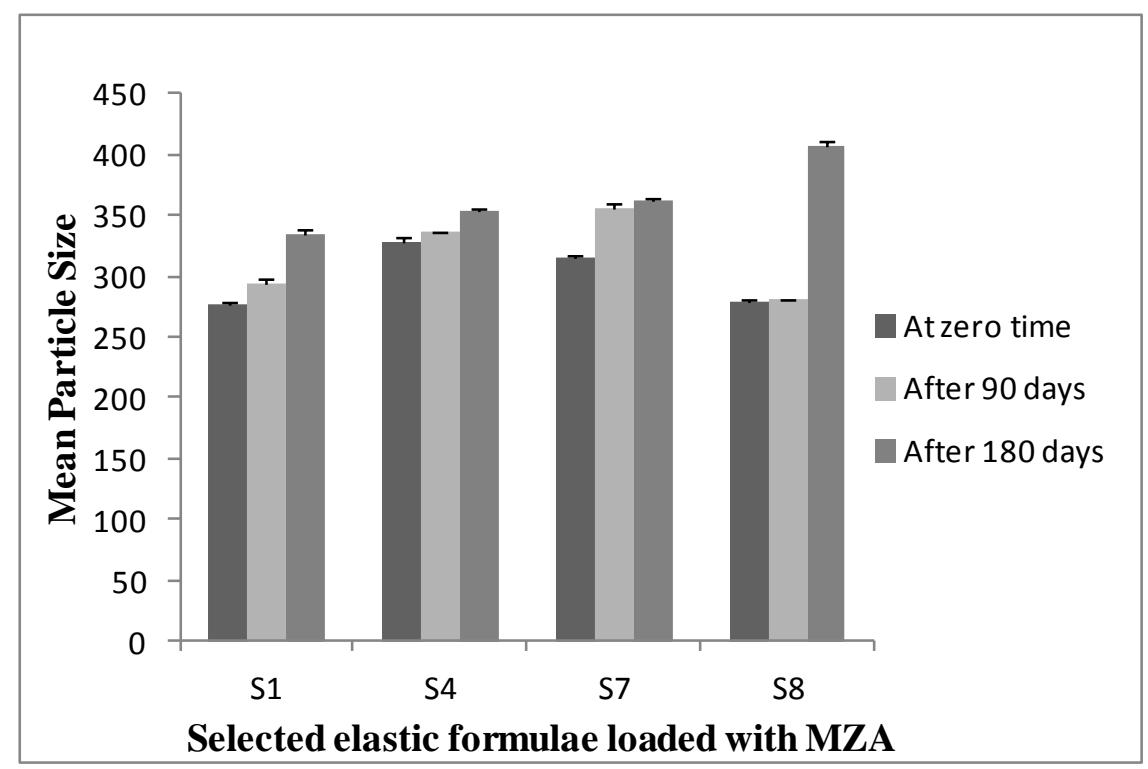

Fig 4: Mean PS of elastic vesicles after interval of time at $4^{\circ} \mathrm{C}$

\section{CONCLUSION}

MZA was encapsulated successfully in elastic vesicles prepared by mixing different ratios of Span 60: edge activators. The best formulae that have smallest PS, and highest EE \%, were selected for further investigations. The formulae showed delayed drug release when compared with drug solution.

\section{REFERENCES}

Abdallah, M; Sammour, O; EL-Ghamry, H; and Abu-Selem, M; (2013): Preparation and in-vitro evaluation of diclofenac sodium niosomal formulations. IJPSR .4,1757-1765.

Akhilesh, D; Bini, K; and Kamath, J; (2012): Review on Span-60 Based Non-Ionic Surfactant vesicles Niosomes as Novel Drug Delivery. International Journal of Research in Pharmaceutical and Biomedical Sciences 3 (1).

Attama, A.A: Reichl, S; and Müller- Goymann, C.C; (2008): Diclofenac sodium delivery to the eye: in vitro evaluation of novel solid lipid nanoparticle formulation using human cornea construct. Int J Pharm 355, 307-313.

Basha, M; Abd El-Alim, SH; Shamma, RN; and Awad, GE; (2013): Design and optimization of surfactant-based nanovesicles for ocular delivery of Clotrimazole. J Liposome Res 1-8.

Chen, R; Qian, Y; Li , R; Zhang, Q; Liu, D; Wang, M; and Xu, Q; (2010): Methazolamide calcium phosphate nanoparticles in an ocular delivery system. Yakugaku Zasshi 130,419-424.

Dash, S; Murthy, P.N; Nath, L; and Chowdhury, P; (2010): kinetic modeling on drug release from controlled drug delivery systems acta poloniae pharmaceutica drug research, 67, 217- 223.

Edelhauser, HF; and Maren, TH; (1988): Permeability of human cornea and sclera to sulfonamide carbonic anhydrase inhibitors. Arch Ophthalmol 106,11101115 . 
El Zaafarany, GM; Awad, GA; Holayel ,SM; and Mortada, ND; (2010): Role of edge activators and surface charge in developing ultradeformable vesicles with enhanced skin delivery. International Journal of Pharmaceutics 397: 164172.

Fridriksdóttir, H; Loftsson, T; and Stefánsson, E; (1997): Formulation and testing of methazolamide cyclodextrin eye drop solutions. Journal of Controlled Release 44, 95-99.

Guinedi, A.S; Mortada, N.D; Mansour, S; and Hathout, R.M; (2005): Preparation and evaluation of reverse-phase evaporation and multilamellar niosomes as ophthalmiccarriers of acetazolamide. Int. J. Pharm. 306, 71-82.

Hathout, RM; Mansour, S; Mortada, ND; and Guinedi, AS; (2007): Liposomes as an ocular delivery system for acetazolamide: in vitro and in vivo studies. AAPS Pharm Sci Tech 8,1.

Huang, Y; Tsai, M; and Wu, P; (2011): Elastic liposomes as carriers for oral delivery and the brain distribution of (+)-catechin. J Drug Target 19:709-718.

Jarvinen, K; Jarvinen , T; and Urtti, A. (1995): Ocular absorption following topical delivery. Advanced Drug Delivery Reviews 16, 3-19.

Kakkar ,S; and Kaur, IP; (2011): Spanlastics A novel nanovesicular carrier system for ocular delivery. International Journal of Pharmaceutics 413:202- 210.

Kaur, IP; Garg, A; Singla, AK; and Aggarwel D. (2004): Vesicular systems in ocular drug deliver: an overview. International Journal of Pharmaceutics 269,1-14.

Lang, J. C. (1995): Ocular drug delivery conventional ocular formulations. Adv. Drug Delivery Rev. 16, 39-43.

Li ,R; Jiang, S; Liu D,.Bi, X; Wang, F; Zhang, Q; and Xu, Q; (2011): A potential new therapeutic system for glaucoma: solid lipid nanoparticles containing methazolamide. Journal of Microencapsulation 28, 134-141.

Lingan, MA; Sathali, AA; and Kumar, MR; (2011): Formulation and evaluation of topical drug delivery System containing clobetasol propionate niosomes. Sci Revs Chem Commun 1, 7-17.

Maren, TH; Haywood, JR; Chapman, SK; and Zimmerman, TJ; (1977): The pharmacology of methazolamide in relation to the treatment of glaucoma. Investative Ophthalmology and Visual Sciences 16, 730-742.

Muller, R. H; Radtke, M; and Wissing; S. A; (2002): Solid lipid nanoparticles (SLN) and nanostructured lipid carriers (NLC) in cosmetic and dermatological preparations. Adv. Drug Deliv. Rev. 54, S131-S155

Nasr, M; Mansour, S; Mortada, ND; and El Shamy, A; (2008): Lipospheres as Carriers for Topical Delivery of Aceclofenac: Preparation Characterization and In Vivo Evaluation. AAPS PharmSciTech 9,154-162.

Padamwar, M.N; Pokharkar, V. B; (2006): Development of vitamin loaded topical liposomal formulation using factorial design approach: Drug deposition and stability. Int. J. Pharm. 320, 37-44.

Patidar and Jain; (2012): Non ionic surfactant based vesicles (niosomes) containing flupirtine maleate as an ocular drug delivery system. Journal of Chemical and Pharmaceutical Research, 10, 4495-4500 
Prabhu, P; Koland, M; Vijaynarayan, K; Harish, Nm; Ganesh, D; Charyulu, Rn; and Satynarayana D; (2010): Preparation and evaluation of niosomes of brimonidine tartrate as ocular drug delivery system. Jprhc 4, 293-301.

Qian, Y; Wang, F; Li, R; Zhang, Q; and Xu, Q. (2010): Preparation and evaluation of in situ gelling ophthalmic drug delivery system for methazolamide. Drug Devlopment and Industrial Pharmacy 36,1340-1347.

Salama, HA; Mahmoud, AA; Kamel, AO; Abdel Hady, M; and Awad GA. (2012): Phospholipid based colloidal poloxamer-nanocubic vesicles for brain targeting via the nasal route. Colloids and Surfaces B: Biointerfaces 100:146-154.

Shahiwala, A; Misra, A; (2002): Studies in topical application of niosomally entrapped Nimesulide. J. Pharma. Pharmaceut. Sci. 5, 3, 220-225.

Van den Bergh, BA; Bouwstra, JA; Junginger, HE; and Wertz, PW; (1999): Elasticity of vesicles affects hairless mouse skin structure and permeability. J Control Release 62:367-79.

Van den Bergh, BA; Wertz, PW; Junginger, HE; and Bouwstra, JA; (2001): Elasticity of vesicles assessed by electron spin resonance, electron microscopy and extrusion measurements. Int J Pharm 217:13-24.

Wilson, B; Samanta, M; and Santhi, K; (2008): Poly(n-butylcyanoacrylate nanoparticles coated with polysorbate 80 for the targeted delivery of rivastigmine into the brain to treat Alzheimer's disease. Brain Res 1200:159-168.

Yoshioka, T; Sternberg, B; and Florence, AT; (1994): Preparation and properties of vesicles (niosomes) of sorbitan monoesters (Span 20, 40, 60, 80) and sorbitan triesters (Span 85). Int J Pharm 105:1-6.

Youshia, J; Kamel, A, ; El Shamy, A; and Mansour, S. (2012): Design of cationic nanostructured heterolipid matrices for ocular delivery of methazolamide. International Journal of Nanomedicine 2012-7:2483-2496.

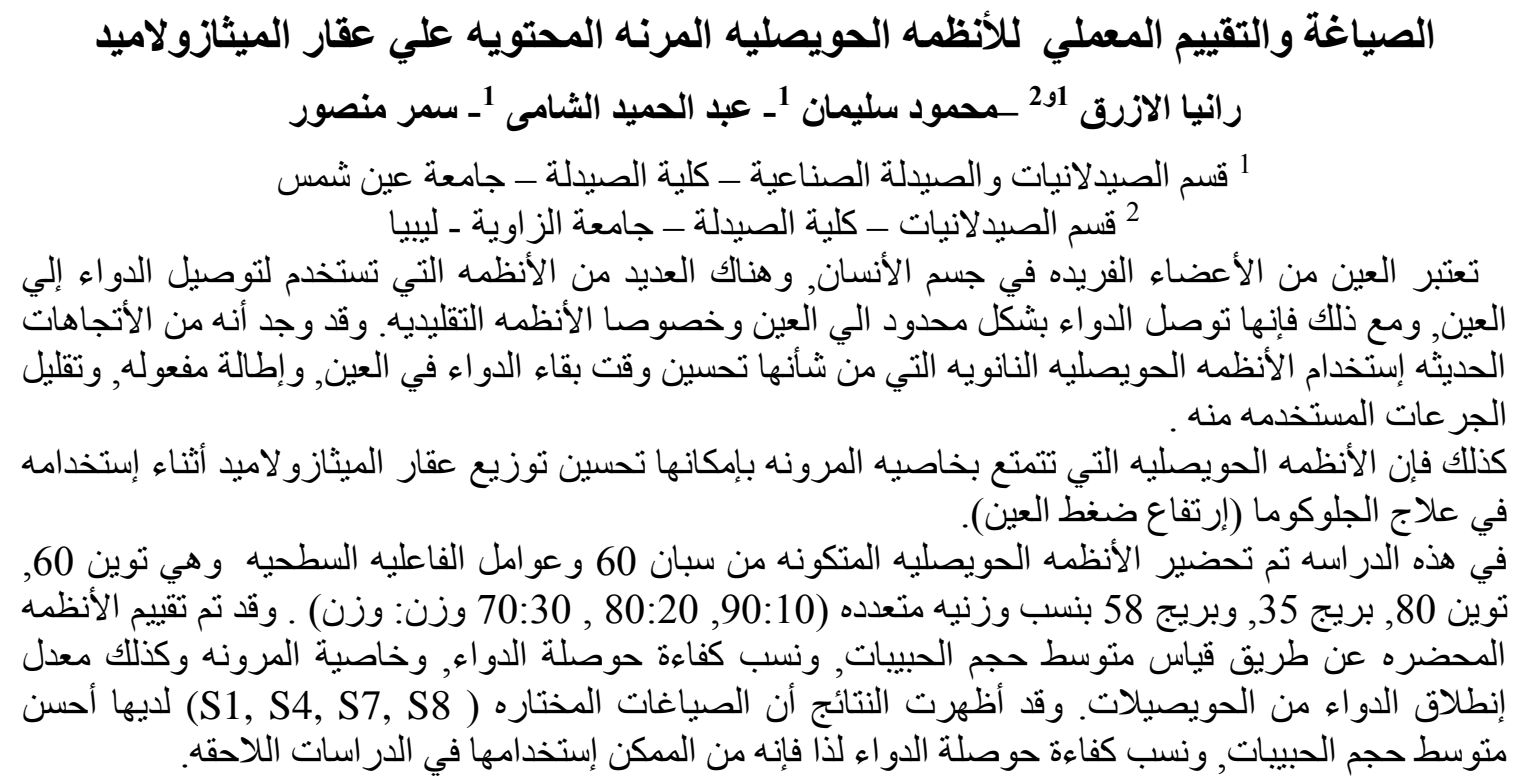

